\title{
Behavioral and Emotional Problems Among Jamaican and African American Children, Ages 6 to II: Teacher Reports Versus Direct Observations
}

\author{
Marieva Puig, Michael Canute Lambert, George T. Rowan, Tyrone Winfrey, \\ Mikhail lyubansky, Stanley D. Hannah, and Martin F. Hill
}

$\mathrm{R}$ ESEARCHERS IN THE FIELD OF DEvelopmental psychopathology have recognized the importance of building a database on children's behavioral and emotional problems prevalent in diverse nations. Several studies have compared U.S. children's problems with those of children in other regions of the world, including the Caribbean (e.g., Lambert, Knight, Taylor, \& Achenbach, 1996), Europe (e.g., Achenbach, Verhulst, Baron, \& Akkerhuis, 1987), Asia (e.g., Weisz et al., 1987), Africa (e.g., Weisz, Sigman, Weiss, \& Mosk, 1993), and Australia (Achenbach, Hensley, Phares, \& Grayson, 1990). Most of these studies consist of reports from parents and teachers who are personally involved with the children. Although information from parents and teachers is valuable, their reports may be influenced by the culturally linked values and expectations of what they consider typical behavior (Weisz, Chaiyasit, Weiss, Eastman, \& Jackson, 1995). Therefore, it is possible that cross-national findings reflect parents' and teachers' expectations regarding children's behavior rather than the actual problems that children present.

Several studies have demonstrated the importance of comparing different informants' (i.e., teachers, parents, observers) reports of children's behavior. A study comparing Jamaican and U.S. teacher reports on children ages 6 to II revealed that Jamaican teachers reported higher problem scores in their pupils than U.S. teachers. Do Jamaican children really have more problems than U.S. children, or do teachers in these two countries have different tolerance levels for certain problems? This study addressed this question by comparing observers' and teachers' reports on Jamaican and African American children. We obtained teachers' reports and conducted structured direct observations on the same group of children. Considerable disparity emerged between observers' and teachers' ratings of Jamaican versus African American pupils. The findings suggest that ethnic similarities between Jamaican pupils and their teachers and the lack of similarities between African American pupils and their teachers may affect the teachers' threshold for perceiving the problems that their pupils present.

A study with Thai and U.S. children that compared teacher reports with structured observations revealed very different patterns in children's emotional and behavioral problems based on the interaction of nationality and reporter (i.e., observer versus teacher; Weisz et al., 1995). Thai teachers reported levels of problem behavior for Thai children that were twice as high as their U.S. peers reported for their pupils. In contrast, structured observations of Thai and U.S. children's school behavior revealed twice as many emotional and behavioral problems in U.S. children than in their Thai age-mates. These findings suggested that teachers' thresholds for child prob- lems varied across the two nations and that teachers' reports were heavily influenced by culture-linked values and expectations about appropriate behavior in school.

The Thai-U.S. multi-informant study addressed a critical aspect of informant bias in teachers' ratings and underscored the need to incorporate information from different sources. However, no other study has examined these issues in the United States and other Western nations such as Jamaica. Addressing these issues in Jamaica and the United States can add to the growing body of research that has compared children's problems across these two nations (e.g., Lambert, Lyubansky, \& Achenbach, 1998). 
Like other cross-national studies on children's problems (Weisz et al., 1988), comparisons between Jamaican and U.S. children have been primarily based on reports made by parents and teachers. An epidemiological survey of children's problems reported by parents in Jamaica and the United States found few significant differences between problems reported for Jamaican versus U.S. children (Lambert, Knight, Taylor, \& Achenbach, 1994). However, a recent study comparing teacher-reported behavior problems of Jamaican and U.S. children in the same age group (i.e., children ages 6 to 11) revealed that Jamaican teachers reported higher total problem scores than U.S. teachers (Lambert et al., 1996). Like their Thai colleagues (Weisz et al., 1988), Jamaican teachers rated their pupils as having higher problem scores than U.S. teachers regardless of age and gender. These findings may, in part, indicate that Jamaican teachers may have different thresholds of distress regarding child problems than do U.S. teachers. It is possible that teacher's ratings across the two nations do not reflect actual differences in school-based problems between Jamaican and U.S. children but reflect cross-national biases in teacher ratings.

Another factor that may account for these findings is the heterogeneous ethnic group composition within and across the samples surveyed. Although the Jamaican sample was primarily of African descent and the U.S. sample primarily of European descent, the earlier Jamaican versus U.S. comparisons did not directly address issues associated with ethnicity. Like the earlier JamaicanU.S. surveys, studies that combine ethnic groups may obscure findings due to ethnicity (Lambert, Puig, Rowan, Lyubansky, \& Winfrey, 1998; Malgady \& Rogler, 1998).

The absence of a direct focus on children of African descent in the Jamaican-U.S. cross-national studies reflects a major problem in behavioral science research. Researchers seldom focus specifically on children of color (McLoyd, 1995). Research that focuses on children of similar heritage (e.g., African diaspora) who reside in different societies has the potential to determine which problems are heritage based and which are more likely to be environmentally based. For example, problems that are similar across different societies may suggest the need for an exploration that focuses on whether these are heritage based. By contrast, problems that differ across groups may indicate that the environment is a contributing factor. Research findings that lead to the identification of contributing factors regarding children's problems in either or both societies can inform policy and intervention decisions to ameliorate these problems. Comparisons of Jamaican and African American children can provide this opportunity.

Like African American children, Jamaican children are predominantly descendants of West Africans who were enslaved by Europeans. Although both sets of children may be subjected to childrearing practices that reflect AfricanBritish ethos (e.g., respect for authority figures), Jamaicans of African descent are the majority group in their country (Lambert, Knight, et al., 1994). By contrast, their African American peers form the largest minority group in the United States. Thus, despite their similarity in heritage, children of African descent in each country are placed in very different school-based situations. For example, Jamaican children are almost always educated by teachers from their own ethnic group, whereas African American children are usually educated by teachers from other ethnic groups, and especially by teachers of European descent (Lambert, Puig, et al., 1998). The value systems and thresholds toward behavioral problems of teachers who are from the same socioethnic background as their pupils may vary considerably from those of teachers that are not from similar backgrounds. For example, one study found that teachers' assessments of students were influenced by cultural differences between the teacher and the student (Zimmerman, Khoury, Vega, Gil, \& Warheit, 1995). Thus, one must be cautious when making inferences from comparison studies of U.S. versus Jamaican teacher ratings (e.g., Lambert et al., 1996) that grouped chil- dren from different ethnic backgrounds together.

This study extends previous research on comparisons of Jamaican and U.S. teachers' reports on child problems. Furthermore, it addresses the problems identified in previous comparisons in two important ways. First, it incorporates the methodology of structured direct observations of emotional and behavioral problems in both countries. Second, it focuses entirely on children of African descent in both countries. The goals of this study are (a) to use the direct observation methodology to determine the nationality-linked similarities and differences between Jamaica and the United States in the problems that children of African descent exhibit in classroom settings and (b) to document whether the number of total problems, the specific syndromes (e.g., anxious/ depressed, aggressive behavior), and the problem types (i.e., internalizing vs. externalizing groupings of the syndromes) reported, vary according to informant (teacher vs. observer) and nationality (Jamaican vs. African American) for the two groups of children of African descent.

\section{Method}

\section{Sample}

The sample included 102 children ages 6 to 11 , with an overall mean age of 9.0 $(S D=1.12)$. Of the total sample, 54 children were Jamaicans of African descent and 48 were African American children from Michigan. Children were selected from elementary schools in Jamaica and Michigan. The Jamaican sample consisted of 27 boys and 27 girls, and the African American sample consisted of 24 boys and 24 girls. Of the Jamaican sample, 95\% were of African descent; other groups, mixed with African and East Indian, made up the remaining $5 \%$. The U.S. sample was $94 \%$ African American; $6 \%$ were of mixed descent (i.e., African American and Hispanic or African American and European American). All teachers in Jamaica were of African descent. In the United States, $7(29 \%)$ of the teachers were African 
American, $16(67 \%)$ were European American, and 1 (4\%) was Hispanic.

Children were randomly selected from multiple elementary schools in each country. In Jamaica, 54 children were sampled from three schools in urban areas of Kingston, the largest city in Jamaica. These schools included two public schools and one private school. To match the Jamaican urban sample, the U.S. sample consisted of 48 children drawn from three urban elementary schools in two cities in middle and eastern Michigan. Similar to the Jamaican sample, the U.S. schools included two public schools and one private school.

For Michigan children, we scored socioeconomic status (SES) according to Hollingshead's (1975) 9-step scale for the parent having the higher status occupation, where 9 is the highest. The U.S. mean SES was 4.1 ( $S D=1.9)$. For Jamaican children, we used a 5-step SES scale (Smith, 1984) designed specifically for Jamaicans. The mean score for Jamaican children was $2.2(S D=0.98)$. The scores derived from the Jamaican SES scale were divided into the following three categories: lower SES $=1.0$ to 2.0 ; middle $\mathrm{SES}=2.5$ to 3.5 ; upper $\mathrm{SES}=4.0$ to 5.0. For calibration with the Jamaican SES scale, Hollingshead's 9-step scale was also divided into three categories: lower SES $=1.0$ to 4.0 ; middle $\mathrm{SES}=4.5$ to 6.5 ; upper $\mathrm{SES}=7.0$ to 9.0. Half steps (e.g., 3.5) on both Jamaican and U.S. SES scales reflect the fact that the occupations that were not clearly scorable were given the mean of the two scores that seemed most appropriate. Previous comparisons of SES for Jamaican and U.S. children on both Smith's and Hollingshead's scales calibrated the scales accordingly and yielded high correlations (e.g., $r=.90$; Lambert, Knight, et al., 1994) between children who were rated on both scales. The present study, therefore, adopted procedures that successfully used Smith's and Hollingshead's SES classifications in cross-national studies involving Jamaican and U.S. youngsters (e.g., Lambert, Lyubansky, \& Achenbach, 1998).

Because numerous studies have documented that SES is negatively correlated with behavior and emotional prob- lems (e.g., Dohrenwend et al., 1992), we included SES as a covariate in our model and, thus, tested and controlled for SES effects in all our analyses. As with earlier Jamaican and U.S. comparisons, we used the converted 3-step SES rating scales in each nation.

\section{Observational Procedures and Measures}

Similar procedures were used to collect the Jamaican and African American sample. The Jamaican sample was collected during the summer of 1996, and the African American sample was collected during the summer of 1997. In the three elementary schools in Jamaica and the three schools in Michigan, we randomly selected children in Grades 1 through 6. Although we observed only two children in each class, we selected every third child on the class list (i.e., Child 3, 6, 9, etc.). Each selected child was asked to take home a consent form asking parents for their child's participation in the study. We chose this methodology to prevent the randomly selected participant from knowing that he or she was chosen to be observed. We asked parents of Jamaican and African American children for permission to observe their child during regular classroom activities and to record these observation ratings on the Direct Observation Form (DOF) of the Child Behavior Checklist (Achenbach, 1986). We also asked parents' permission for their child's teacher to complete the Teacher's Report Form of the Child Behavior Checklist (TRF; Achenbach, 1991). Once parental permission was received, we randomly selected one boy and one girl from a list of children with parental consent, with the constraint that we maintained an age balance.

Direct Observation Form. The DOF consists of 97 individual problem behavior items (e.g., makes odd noises, argues, teases, physically isolates from others) on which observers rate children on a 4-point scale of 0 (not observed), 1 (slight or ambiguous occurrence), 2 (definite occurrence with moderate intensity and less than 3 minutes duration), or 3 (definite occurrence with strong inten- sity or greater than 3 minutes duration). In addition, the DOF includes on/offtask ratings. At the end of every minute, the observer checks off whether or not the child was on task.

Principal components analyses on the DOF have yielded six syndromes labeled Withdrawn-Inattentive, NervousObsessive, Depressed, Hyperactive, Attention Demanding, and Aggressive. Second-order factor analyses have yielded two major groupings of these syndromes, internalizing and externalizing (Reed \& Edelbrock, 1983).

Each child was observed four different times within a 2-week period. Each rated observation period lasted $10 \mathrm{~min}$ utes. Observation times were randomly selected from regular class times. Lunch and recess periods were excluded. Each child was observed throughout the day, morning and afternoon. Reliability information on observational procedures was obtained by calculating agreement between trained observers using intraclass correlation coefficients (ICCs).

Intraclass Correlations. The Jamaica observer team consisted of four observers (one Caribbean and three African Americans) who had received their training in psychology in the United States. These observers were temporarily living in Jamaica and thus were familiar with Jamaican idioms, culture, and customs. Three of these observers also collected data in the United States and served as reliability standards for both countries. These three observers were graduate students in clinical and counseling psychology. The U.S. observer team included the three observers serving as reliability standards and five other observers. The additional observers of the U.S. team consisted of one Caribbean, one Asian, and three European Americans. Fifteen randomly selected children (from Jamaica and the United States) from the total sample of 102 children were observed for reliability purposes. Interobserver reliabilities were calculated using ICC between pairs of observers. The average total problem score (i.e., the sum of the ratings across all 97 problem items on the DOF) ICC for the observers was .84 . The average 
ICC for internalizing problems (e.g., nervous, withdrawn) was .57 ; for externalizing problems (e.g., loud, swears), it was .73. For the on/off-task score, the ICC average was .89. Internalizing problems reflect an internal state of the child. Thus, the reporter or observer must make more subjective judgments regarding this type of behavior than for externalizing behavior. The indices reflecting lower rates of agreement for internalizing problems may therefore reflect differences in subjective judgments.

Teacher Report Procedures and Measures. Similar procedures were used to collect teacher reports in both countries. We asked each parent of African American and Jamaican children to allow their child's teacher to complete the Child Behavior Checklist-Teacher's Report Form (TRF; Achenbach, 1991) or the Jamaican Teacher's Report Form (JTRF; Lambert, Knight, \& Costigan, 1994), respectively. All teachers completed two Teacher's Report Forms, one for each girl and each boy observed in their classroom. A total of 27 teachers of Jamaican children and 24 teachers of African American children were asked to complete the checklists. All teachers consented to complete the Teacher's Report Forms.

U.S. Teacher's Report Form. Teachers in the United States completed the Child Behavior Checklist-Teacher's Report Form (TRF; Achenbach, 1991). The TRF includes demographic information, questions about the child's adaptive functioning and academic performance, standardized test scores, and a list of 118 specific problems. Based on the preceding 2 months, teachers scored each problem as 0 (not true of the child), 1 (somewhat or sometimes true of the child), or 2 (very true or often true of the child). Test-retest reliability for total problem scores was $r=.95$ for a mean interval of 15 days across various groups of children. The interrater correlation between teachers was $r=.60$ for total problems (Achenbach, 1991). Principal component analyses of the TRF have yielded eight syndromes, labeled Withdrawn, Somatic Complaints, Anxious/ Depressed, Thought Problems, Attention
Problems, Delinquent Behavior, Social Problems, and Aggressive Behavior. Second-order principal factor analyses have yielded internalizing and externalizing groupings of the syndromes.

Jamaican Teacher's Report Form. Teachers in Jamaica completed the Jamaican version of the TRF, designated as the Jamaican Teacher's Report Form (JTRF). The JTRF has the same format as the TRF. Demographic items, child's adaptive functioning, and academic items are followed by problem items using the same 3-point rating scale, all in the same order as on the TRF. Some of the items on the JTRF are slightly modified to represent Jamaican idiomatic expressions. In addition, the JTRF contains 32 extra items with problems particularly relevant to Jamaican children. However, to facilitate cross-national comparisons, only the items common to both instruments were included in the analyses. As an estimate of test-retest reliability, a mean ICC of .78 was obtained for problem scores derived from 20 teachers who completed the JTRF twice over a 1-week interval. Interrater agreement among 20 Jamaican teacher pairs who independently rated the same pupils in different conditions revealed an ICC of .61 (Lambert et al., 1996).

\section{Classroom Conditions in Both Countries}

The classroom conditions in Jamaica were different from those in the United States. In Jamaica, there was an average of 40 students per class in public schools and 25 children per class in private schools. In contrast, U.S. classes contained an average of 28 children in public schools and 15 children per class in private schools. Private schools in both countries contained less children per classroom. However, Jamaican classrooms were still more crowded compared to U.S. classrooms.

The classroom settings in each country also varied. In Jamaican schools, children sat in benches, which seated about three children. Moreover, Jamaican classrooms were usually separated only by a row of blackboards indicating the division between the classrooms. Thus, the activities from one classroom could easily be heard and seen from other classrooms. Notably, although Americans may perceive this classroom arrangement as distracting to the pupils, Jamaican teachers' authority was such that discipline prevailed in the classrooms. This classroom setting differs from that of U.S. elementary schools, in which each child has his or her own desk and each classroom is separated by a solid wall.

\section{RESULTS}

\section{Data Analyses}

To limit the chance of Type I error, we used the Bonferroni correction (Cliff, 1987; Neter, Wasserman, \& Kutner, 1985). The Bonferroni correction adjusts the alpha level to take into account the number of statistical tests performed and, thus, sets the alpha level at .006 for the first wave of analyses and .004 for the second wave. We interpreted the sizes of the significant effects using Cohen's (1988) criteria. Cohen classifies analysis of covariance (ANCOVA) effect sizes (ES) as small if they account for $1.0 \%$ to $5.9 \%$ of the variance, medium if they account for $5.9 \%$ to $13.8 \%$, and large if they account for more than $13.8 \%$.

In all analyses, we entered SES and age as continuous variables in the model to simultaneously control for and test their effects-that is, the analyses were based on an ANCOVA model with SES and age as covariates. Therefore, all means reported are adjusted means (i.e., via a least square means procedure) to reflect the effects of the covariates.

The first wave of analyses focused on the observational measures using a 2 (nationality) $\times 2$ (gender) ANCOVA with SES and age as covariates. We tested the effects of these variables on total problem scores, on-task scores, each of the six DOF scale scores, and the two broad groupings (internalizing and externalizing) of the scales as dependent variables considered separately. We also tested whether internalizing or externalizing problems were most often observed in 
each country. To achieve this, we performed a 2 (nationality-Jamaican vs. African American) $\times 2$ (gender) $\times 2$ (problem type) repeated measures ANCOVA of the two observational scores, with problem type (internalizing and externalizing) as the within-group factor and SES and age as covariates.

The second wave of analyses compared teachers' reports using a 2 (nationality) $\times 2$ (gender) ANCOVA with SES and age as covariates. The total problem scores, each of the eight TRF syndromes, and the two broad groupings of the syndromes (internalizing and externalizing) were dependent variables considered separately. Similar to the analyses involving observers' reports, we also tested for teacher-reported problem type differences across each country. Thus, we performed a 2 (nationality) $\times 2$ (gender) $\times 2$ (problem type) repeated measures ANCOVA on teacher reports, with problem type as the within-group factor and SES and age as covariates.

Finally, teachers' versus observers' reports were analyzed using only the 86 items that were common to both the teacher form and the observation form. To have comparable scores for the Teacher Form (0-2 scale) and the observation measure ( $0-3$ scale), the 4 -point observation measure was first converted to a 3-point scale by multiplying scores by two thirds. Next, teachers' versus observers' ratings were analyzed via a 2 (reporter-teacher vs. observer) $\times 2$ (nationality) $\times 2$ (gender) repeated measures ANCOVA, with reporter as the repeated measure factor and SES and age as covariates. We conducted separate analyses for total problem scores and for internalizing and externalizing problems as dependent variables.

\section{Observer Results}

\section{On/Off-Task Scores}

A $2 \times 2$ (nationality $\times$ gender) ANCOVA of on-task scores, with SES and age as covariates, yielded a main effect of nationality, $F(1,96)=9.68, p=.0025$. This effect accounted for $9.9 \%$ of the variance in on-task scores, a medium ES. African American children were on task an average of 8 minutes $(S D=0.10)$ out of each 10-minute observation period. Jamaican children were on task an average of 6.5 minutes ( $S D=0.14$ ) out of each 10-minute observation period (see Table 1).

\section{Total Problem Scores}

The $2 \times 2$ (nationality $\times$ gender) ANCOVA, with SES and age as covariates, yielded a nationality main effect, $F(1,96)=13.27, p=.0005$, on total problem scores with a medium ES accounting for $10 \%$ of the variance. Table 1 indicates that the mean observation total problem score for African American children was significantly lower than that for their Jamaican peers. Thus, observers recorded more problems for Jamaican than African American chil- dren. The nationality main effect was moderated by a significant age $\times$ nationality interaction, $F(1,96)=7.74$, $p=.006$. This interaction effect accounted for $5.7 \%$ of the variance of total problem scores, a small ES. Breaking down the interaction into its components, we found that observers rated younger Jamaican children as exhibiting more total problems than older children, $F(1,96)=10.39, p=.0019$, whereas for African American children, the effect of age was nonsignificant for total problem scores.

\section{Syndromes, Internalizing and Externalizing Problems}

Significant nationality main effects emerged for Withdrawn/Inattentive, Hyperactive and Aggressive scales, $F(1$,

\begin{tabular}{|c|c|c|c|c|}
\hline \multicolumn{5}{|c|}{$\begin{array}{c}\text { TABLE I } \\
\text { Observer Report and Teacher Report Measures for } \\
\text { Jamaican and African American Children }\end{array}$} \\
\hline \multirow[b]{2}{*}{ Syndrome } & \multicolumn{2}{|c|}{ Jamaican children } & \multicolumn{2}{|c|}{ African American children } \\
\hline & $M$ & SD & $M$ & $S D$ \\
\hline Observers & & & & . \\
\hline On task & $6.50^{*}$ & 0.14 & $8.00 *$ & 0.10 \\
\hline Total problems & $15.29 *$ & 4.10 & $9.37^{*}$ & 3.30 \\
\hline Internalizing & $5.63^{*}$ & 3.00 & $3.60^{*}$ & 2.60 \\
\hline Externalizing & 3.01 & 1.80 & 2.20 & 1.30 \\
\hline Withdrawn-inattentive & 4. $10^{*}$ & 2.20 & $2.50^{*}$ & 1.10 \\
\hline Nervous-obsessive & 1.70 & 0.99 & 1.02 & 0.91 \\
\hline Depressed & 1.30 & 0.94 & 1.10 & 0.91 \\
\hline Hyperactive & $6.60^{*}$ & 2.50 & $3.70^{*}$ & 2.10 \\
\hline Attention demanding & 0.89 & 0.56 & 1.15 & 0.84 \\
\hline Aggressive & $2.40^{*}$ & 1.90 & $1.40^{*}$ & 0.99 \\
\hline \multicolumn{5}{|l|}{ Teachers } \\
\hline Total problems & $26.60^{*}$ & 20.60 & $44.30^{*}$ & 24.40 \\
\hline Internalizing & 8.17 & 1.30 & 8.81 & 2.50 \\
\hline Externalizing & $6.55^{* *}$ & 2.20 & $18.90^{* *}$ & 5.20 \\
\hline Withdrawn & 3.47 & 2.19 & 2.90 & 1.40 \\
\hline Somatic complaints & 0.41 & 1.90 & 1.20 & 0.76 \\
\hline Anxious-depressed & 4.70 & 2.10 & 5.10 & 2.30 \\
\hline Thought problems & 0.74 & 0.58 & 0.83 & 0.63 \\
\hline Attention problems & 9.40 & 4.61 & 11.78 & 5.31 \\
\hline Delinquent behavior & $1.16^{*}$ & 0.96 & $3.00^{*}$ & 2.00 \\
\hline Aggressive behavior & $5.38 * *$ & 2.20 & $15.93 * *$ & 5.10 \\
\hline Social problems & 1.64 & 0.83 & 3.74 & 1.90 \\
\hline
\end{tabular}

$*_{p}<.005 . *_{p}<.0001$. 
96) $=8.39, p<.004$. Table 1 shows that for all the significant effects Jamaican children received higher mean observer ratings than did African American children. All ES were medium.

Similar effects emerged for internalizing scores. As noted in Table 1, Jamaican children received higher observer ratings than African American children, $F(1,96)=4.17, p=.004, \mathrm{ES}=10 \%$, a medium ES. Although we tested for problem-type differences (as a withinsubjects factor) in a repeated measures ANCOVA, this analysis revealed no significant interaction or main effects.

\section{TEACHER Results}

\section{Total Problem Scores}

The 2 (nationality) $\times 2$ (gender) ANCOVA yielded a significant nationality main effect for total problem score, $F(1,96)=9.36, p=.0029$, with a medium ES accounting for $8.9 \%$ of the variance. Table 1 indicates that teachers in the United States reported significantly higher total problem scores for African American children than teachers in Jamaica reported for their pupils. No significant interactions emerged.

\section{Syndromes, Internalizing and Externalizing Problems}

Significant nationality effects were found for two of the eight syndromes: Delinquent Behavior, $F(1,96)=12.91$, $p=.0005$, accounted for $12 \%$ of the variance, a medium $E S$, and Aggressive Behavior, $F(1,96)=21.98, p=.0001$, accounted for $18 \%$ of the variance, a large ES. As shown in Table 1, U.S. teachers rated African American children as having significantly higher Delinquent Behavior and Aggressive Behavior scores than their Jamaican colleagues indicated for their pupils.

No significant effects were found for internalizing problems. A significant nationality main effect was found for externalizing problems, $F(1,96)=$ $21.70, p=.0001$. This effect accounted for $17 \%$ of the variance, a large ES. Teachers rated African American chil- dren as having significantly more externalizing problems than Jamaican teachers rated their children. When internalizing versus externalizing problems were compared using repeated measures ANCOVA with problem type as the within-group factor, no significant within- or between-group main effects emerged. There were also no significant within- or between-group interactions.

\section{Observational Data Versus Teacher Reports}

As previously noted, the DOF and both the Jamaican and the U.S. versions of the TRF have 86 overlapping items. These items allowed us to compare total problem score ratings across informants. Therefore, we performed a repeated measures ANCOVA with gender and nationality as independent variables, SES and age as covariates, total problem score as the dependent variable, and reporter as the within-subjects factor. The analyses revealed a significant reporter within-subjects main effect, $F(1,94)=23.43, p=.0001$, for total problems. This effect accounted for $5.7 \%$ of the variance, a small ES. Teachers' ratings were higher than observers' ratings. Means for teachers and observers across both countries were 25.5 $(S D=19.6)$ and $10.0(S D=2.0)$, respectively.

In addition to the within-subjects main effect, there was a significant reporter $\times$ nationality within-subjects interaction, shown in Figure 1, $F(1,94)$ $=7.3, p=.0072$. This effect accounted for $1.7 \%$ of the variance of total problems, a small ES. Breaking the interaction into its respective components revealed that teachers in the United States rated their African American pupils ( $M=34.47, S D=14.5)$ as having significantly higher total problems scores than Jamaican teachers rated their pupils $(M=19.32, S D=10.1), F(1,94)=$ $28.3, p<.0001$, with a large ES accounting for $15 \%$ of the variance. By contrast, observers' ratings showed a significant effect in the other direction, with Jamaican children receiving significantly higher ratings on total problems $(M=13.65$, $S D=2.9$ ) than African American chil- dren $(M=7.2, S D=3.1), F(1,94)=$ $17.37, p<.0001$, with a medium ES accounting for $11 \%$ of the variance.

Viewing the interaction from the other direction, in Jamaica, teachers rated their pupils as having significantly higher total problems scores than observers' ratings of the same children, $F(1,94)=10.41, p<.001$. This was a small ES, accounting for $3 \%$ of the variance. In the United States, teachers' ratings of African American children's total problem scores were also significantly higher than observers' ratings of these African American children, $F(1,94)=$ $33.22, p=.0004$. However, this was a large ES, accounting for $16 \%$ of the variance.

In addition to these analyses, correlational analyses between teachers' and observers' ratings of total problems were conducted for each country. As expected from the previous findings, teachers' and observers' ratings had low correlations, and all were nonsignificant.

\section{Most Common Problems Reported by Teachers and Observers}

On a more qualitative note compared to the previous quantitative analyses, Table 2 and Table 3 list the 10 most frequently endorsed (i.e., being present-rating of 1 or more) problems for Jamaican and African American children, based on our observational data and teachers' reports. These qualitative data indicate that teachers' reports in the two countries reflect differences in the types of problems that teachers endorsed. For example, 7 of the 10 most common problems that U.S. teachers endorsed as present in their pupils were externalizing types of problems (see Table 2).

By contrast, 7 of the 10 most common problems than Jamaican teachers endorsed for their pupils were other problems that did not load on either externalizing or internalizing groupings. We also conducted chi-square tests of association on the 10 most frequently noted item as reported by teachers and observers in each country. To decrease the chances of Type I error, scores were compared to an adjusted alpha of .001 . 


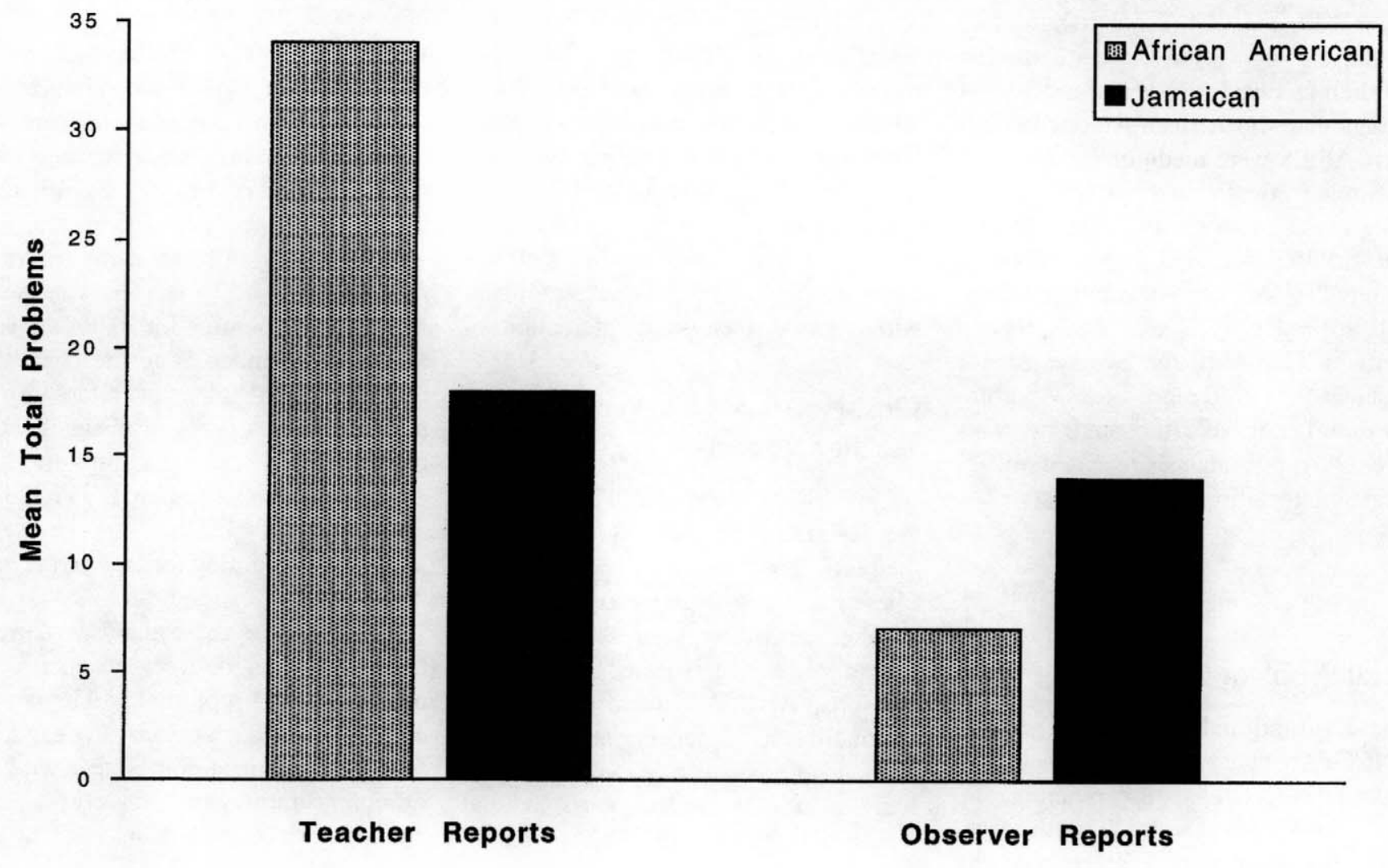

FIGURE I. Mean ratings of total problem scores of African American and Jamaican children, as reported by teachers and trained observers. These are mean comparisons of only the 86 similar items across the TRF and DOF measures.

Tables 2 and 3 also list the results of these analyses, which indicated that, of the 10 most common problems reported by U.S. teachers, significant effects emerged for 7 items. These effects indicated that significantly larger proportions of U.S. teachers endorsed these problems for African American children than Jamaican teachers did for their pupils. For the 10 most common problems endorsed by Jamaican teachers, a significantly larger proportion of Jamaican teachers endorsed these problems for only 3 out of the 10 items.

Focusing on the observers' ratings, the results indicated that, of the 10 most common problems observers endorsed for African American children, significant effects emerged for 2 . For the items "fidgets with objects" and "slow/lacks energy," observers endorsed these problems in a significantly larger proportion of African American children than Jamaican children. By contrast, of the 10 most common problems observers endorsed as present among Jamaican children, significant effects emerged for 8 items. Observers noted a significantly larger proportion of these problems (see Table 3) for Jamaican children than for African American children. Higher observer ratings emerged for African American children on one item, "fidgets with objects."

\section{Discussion}

Teachers' versus observers' ratings of African American and Jamaican children's behavioral and emotional problems in the classrooms yielded different cross-national patterns. Similar to previous Jamaican-U.S. teacher report comparisons that involved a mixture of several different ethnic groups in the U.S. sample (Lambert et al., 1996), observers' ratings revealed higher total problem scores for Jamaican children than for their African American peers. In con- trast, teachers' ratings indicated that African American children received significantly higher total problem scores than Jamaican children.

The observational methodology used in this study did not allow us to determine reasons for the African American versus Jamaican difference. There may be numerous explanations for this finding, but two possibilities should be considered. First, the numerous problems that observers reported for Jamaican children may result from problems associated with overcrowding and the high pupil/teacher ratio in Jamaican versus U.S. classrooms. In Jamaica, the average pupil/teacher ratio was 45 to 1 , twice as high as the ratio in the average U.S. classroom (22 to 1). Moreover, the small classroom sizes in Jamaica and the limited availability of resources (e.g., seating, privacy across classrooms) in each classroom may have contributed to these problems. As documented elsewhere (Sylva, 1994), poor classroom resources 
TABLE 2

Ten Most Common Problems Among African American and Jamaican Children as Reported by Teachers

\begin{tabular}{|c|c|c|c|c|c|c|}
\hline Item & $\begin{array}{l}\text { Item } \\
\text { no. }\end{array}$ & Type & $\begin{array}{c}\text { African American } \\
(\%)\end{array}$ & $\begin{array}{c}\text { Jamaican } \\
(\%)\end{array}$ & $\chi^{2}$ & $p^{\mathbf{a}}$ \\
\hline \multicolumn{7}{|l|}{ African American children } \\
\hline I. Demands attention & 19 & $E$ & 65.2 & 36.0 & 39.500 & $<.001$ \\
\hline 2. Overtired & 53 & $\mathrm{E}$ & 65.2 & 49.0 & 10.900 & $<.001$ \\
\hline 3. Argues a lot & 03 & $E$ & 63.0 & 44.0 & 16.500 & $<.001$ \\
\hline 4. Disturbs pupils & 24 & $\mathrm{E}$ & 60.9 & 33.0 & 34.700 & $<.001$ \\
\hline 5. Acts without thinking & 41 & 0 & 58.7 & 32.0 & 31.300 & $<.001$ \\
\hline 6. Shows off or clowns & 74 & $\mathrm{E}$ & 58.7 & 21.0 & 70.480 & $<.001$ \\
\hline 7. Does not follow directions & 22 & 0 & 56.5 & 42.0 & 8.000 & ns \\
\hline 8. Talks too much & 93 & $\mathrm{E}$ & 56.5 & 44.0 & 7.400 & ns \\
\hline 9. Cruelty, bullying & 16 & $\mathrm{E}$ & 54.3 & 16.0 & 73.170 & $<.001$ \\
\hline 10. Can't sit still & 10 & 0 & 52.8 & 38.0 & 8.500 & ns \\
\hline \multicolumn{7}{|l|}{ Jamaican children } \\
\hline 1. Hurt when criticized & 81 & 1 & 50.0 & 71.9 & 30.200 & $<.001$ \\
\hline 2. Self-conscious & 71 & 0 & 42.9 & 67.9 & 33.100 & $<.001$ \\
\hline 3. Can't concentrate/pay attention & 08 & 0 & 42.3 & 56.4 & 9.530 & ns \\
\hline 4. Fails to finish things & 04 & 0 & 41.2 & 53.8 & 5.800 & ns \\
\hline 5. Not work to potential & 92 & 0 & 48.5 & 52.6 & .047 & ns \\
\hline 6. Fails to do tasks & 100 & 0 & 42.3 & 52.6 & 3.900 & ns \\
\hline 7. Does not follow directions & 22 & 0 & 55.9 & 50.0 & 8.080 & ns \\
\hline 8. Inattentive & 78 & 0 & 51.4 & 50.0 & .150 & ns \\
\hline 9. Talks out of turn & 53 & $\mathrm{E}$ & 64.9 & 34.7 & 10.930 & $<.001$ \\
\hline 10. Messy work & 72 & 0 & 43.5 & 48.7 & 1.040 & ns \\
\hline
\end{tabular}

Note. Numbers in percentage columns indicate the percentage of children for whom nonzero ratings were recorded for the particular item. $I=$ item loaded on Internalizing Problems; $\mathrm{E}=$ item loaded on Externalizing Problems; $\mathrm{O}=$ other problems (i.e., not loading on internalizing or externalizing).

aAlpha adjusted to .001 to decrease change of Type I error.

and high pupil-to-teacher ratio can lead to numerous classroom-based problems. Because the present study did not test for the effects of the number of children per classroom when considering behavioral and emotional problems in the classroom, we cannot be sure that class size was not responsible for the crossnational differences.

Another explanation of the crossnational differences relates to observers' biases. Observers lived in Jamaica for 3 months and became familiar with the Jamaican culture. However, like most professionals who address children's problems in Jamaica, all observers were trained in the United States. Thus, they may have used U.S. standards in rating certain behaviors as problematic, when these behaviors may have been considered typical classroom behaviors by Jamaican teachers.
Shifting the focus to teachers' ratings of African American children, the findings revealed that African American children received total problem score ratings that were almost twice as high as those that Jamaican children received. Moreover, U.S. teachers rated African American children as having significantly more problems on the scales of delinquent and aggressive behavior than Jamaican teachers rated their pupils. These teachers' findings differ from previous research in which Jamaican versus U.S. teachers' ratings of heterogeneous ethnic groups of children revealed more total problems for Jamaican than for U.S. youngsters (Lambert et al., 1996). However, the moderating effect of age on this cross-national observer-reported difference is important, as observers' ratings revealed higher total problem scores in the younger Jamaican sample but teachers' ratings did not. This finding may reflect teachers' versus observers' expectations in Jamaican youngsters. Teachers, like most adults in Jamaica, may view the problems that younger children exhibit as transient, rather than as an enduring trait (Lambert et al., 1992). Thus, the U.S. perspective through which observers viewed the problems presented by Jamaican children may have made the present findings inevitable.

In a similar vein, one must be cognizant of the ethnic and cultural differences and similarities among African American and Jamaican teachers and their pupils. In contrast to the Jamaican cohorts, the teachers of African American pupils were primarily of European American heritage. Their thresholds of tolerance toward problems in African American youngsters may have been 
TABLE 3

Ten Most Common Problems Among African American and Jamaican Children as Reported by Trained Observers

\begin{tabular}{|c|c|c|c|c|c|c|}
\hline Item & $\begin{array}{l}\text { Item } \\
\text { no. }\end{array}$ & Type & $\begin{array}{c}\text { African American } \\
(\%)\end{array}$ & $\begin{array}{c}\text { Jamaican } \\
(\%)\end{array}$ & $\chi^{2}$ & $\mathbf{p}^{\mathbf{a}}$ \\
\hline \multicolumn{7}{|l|}{ African American children } \\
\hline I. Fidgets with objects & 13 & $\mathbf{0}$ & 51.40 & 35.8 & 12.00 & $<.001$ \\
\hline 2. Easily distracted & 56 & $\mathbf{0}$ & 44.20 & 30.8 & 9.42 & ns \\
\hline 3. Doesn't pay attention & 36 & I & 15.80 & 5.9 & 0.02 & ns \\
\hline 4. Slow/lacks energy & 75 & 0 & 32.70 & 6.6 & 29.10 & $<.001$ \\
\hline 5. Stares blankly & 57 & I & 32.00 & 24.0 & 3.80 & ns \\
\hline 6. Doesn't sit still & $\infty$ & 0 & 32.00 & 28.3 & 0.79 & ns \\
\hline 7. Talks too much & 6 & o & 27.00 & 24.0 & 0.44 & ns \\
\hline 8. Disturbs children & 21 & E & 21.00 & 23.16 & 4.80 & ns \\
\hline 9. Sucks thumb, hand & 82 & $\boldsymbol{Q}$ & 11.10 & 14.1 & 0.82 & ns \\
\hline 10. Confused or in a fog & 11 & 1 & 11.00 & 9.7 & 0.23 & ns \\
\hline \multicolumn{7}{|l|}{ Jamaican children } \\
\hline I. Overconforms to rules & 39 & 0 & 21.00 & 34.4 & 3.10 & ns \\
\hline 2. Hurt when criticized & 58 & I & 0.55 & 51.9 & 140.00 & $<.001$ \\
\hline 3. Apathetic, won't try & 44 & 1 & 8.20 & 47.2 & 81.10 & $<.001$ \\
\hline 4. Falls asleep & 43 & 0 & 2.50 & 40.6 & 85.40 & $<.001$ \\
\hline 5. Anxious to please & 78 & $\mathbf{E}$ & 5.50 & 39.7 & 69.40 & $<.001$ \\
\hline 6. Fidgets with objects & 13 & $\mathbf{0}$ & 51.00 & 35.8 & 12.03 & $<.001$ \\
\hline 7. Fearful or anxious & 40 & 1 & 1.10 & 35.8 & 79.10 & $<.001$ \\
\hline 8. Explosive behavior & 54 & 0 & 0.00 & 34.7 & 81.00 & $<.001$ \\
\hline 9. Easily distracted & 56 & 0 & 44.20 & 30.8 & 9.42 & ns \\
\hline 10. Picks nose, skin, etc. & 42 & $\mathbf{0}$ & 25.2 & 30.0 & 0.85 & ns \\
\hline
\end{tabular}

Note. Numbers in percentage column indicate the percentage of children for whom nonzero ratings were recorded for the particular item. $I=$ item loaded on Internalizing Problems; $\mathrm{E}=$ item loaded on Externalizing Problems; $\mathrm{O}=$ other problems (i.e., not loading on internalizing or externalizing).

${ }^{a}$ Alpha adjusted to .001 to decrease change of Type I error.

lower than those of the teachers that were of similar heritage to their pupils (e.g., Jamaican teachers and their pupils). Problem threshold differences may account for the higher problem ratings given by teachers of African American children. These findings match those of earlier United States-based research that compared non-African American teacher ratings of African American youngsters with the ratings that non-Hispanic White and Hispanic teachers gave African American children. Non-Hispanic White and Hispanic teachers rated African American students with higher total problem scores than African American teachers did (Zimmerman et al., 1995). These findings are also reminiscent of a recent study that compared adults' thresholds of tolerance toward emotional and behavioral problems found in African American youth (Lambert, Rowan, et al., 1998). This study revealed that African American parents had very different thresholds toward the problems of African American children depicted in hypothetical vignettes than teachers and clinicians who were not African Americans.

Another important issue to consider is our observers' comments that teachers in Jamaica seemed to be more directly involved in children's lives than teachers in the United States. Observers further noted that the Jamaican teachers were directly involved with the children's families within and outside the school context. This involvement may have a moderating effect on teachers' ratings. For example, Jamaican teachers' knowledge of their pupils' family and home situation and its contribution to their pupils' behavior and emotional adjustment may moderate their ratings and result in lower ratings than those of their U.S. colleagues.
Shifting our focus from the specific findings to the general differences in observers' versus teachers' reports across the two countries, we are tempted to raise the question as to which of the two is more accurate. To address this question, it is necessary to bear in mind the context of the relationship between the reporters and the children surveyed. Data collection on both samples occurred near the end of the academic year. At that time, teachers from both countries had had the opportunity to develop extensive relationships with their pupils. Their ratings, which generally were significantly higher than those of observers, may indicate their lengthy experience with the children and the biases that these experiences engendered. Observers, by contrast, developed no relationship with the children and documented only behaviors that emerged in four separate 10-minute samples. 
However, placing credence in one reporter rather than another may be largely inappropriate, as different reporters provide different perspectives to researchers and clinicians when they study and evaluate children's behavior (Achenbach, 1991). Professionals who desire unbiased ratings on children's classroom behavior may rely more heavily on observer ratings. However, researchers who require a more comprehensive historical perspective-particularly one that may give insight into the relationship between the child and the teacher-may benefit more from teachers' reports. As with other types of information on child assessment, neither is superior to the other, and, in the absence of gold standards regarding child assessment, each informant provides valuable information on children's problems.

One interesting finding was the absence of gender effects on both teachers' and observers' problem scores. One explanation is that this is one of the first studies that has focused particularly on children of the African diaspora in two different countries instead of combining children from different backgrounds into one category of U.S. children. The present findings suggest that gender effects observed in mixed ethnic groups may not be evident for children of the African diaspora who reside in different countries.

Although the findings observed in the present study are intriguing, they should be interpreted in the context of its limitations. First, as we noted earlier, although an appreciable number of our observers resided and worked in Jamaica prior to data collection, most were of U.S. heritage. Thus, despite their training, they may have viewed children's behavior using their U.S. perspective on children's behavior. Second, analyses comparing teachers' and observers' total problem scores should be interpreted with caution because the transformation of scores provided a similar range across measures but did not necessarily equate them. Third, we noted the potential effects of differences in classroom settings, including the pupil-to-teacher ratios, availability of classroom resources, distraction levels from stimuli external to classes (e.g., noise from other classes), and teacher involvement with children and their families. However, we could not measure or control for the contribution of these effects in our findings. Fourth, the samples were drawn from urban areas in both countries and, thus, may not be representative of elementary school children across both countries. Fifth, the size of our sample and the unavailability of African American teachers in the schools we sampled in the United States made it impossible to test teacher ratings according to children's and teachers' ethnicity.

Further research is needed to obtain a larger and more representative sample of children across the two countries and to include more African American teachers. This research can address the concerns regarding sample representativeness and allow testing whether differences or similarities in pupils' and teachers' ethnicity affect teachers' ratings of the problems presented by their pupils. Future research that focuses on issues of observer bias, child-to-teacher ratios in the classroom, availability of basic classroom resources (e.g., seating), distractions (e.g., noise from adjoining classrooms), and teachers' participation in children's lives outside the classroom may elucidate the findings of the present study.

Despite its limitations, this study has provided valuable information on children of African descent who reside in two different countries. It suggests that researchers who assess both sets of youngsters should be mindful of the different perspectives of each reporter. These perspectives may be different and may be colored by the reporter's view of the children's problems. Moreover, these perspectives may not only differ according to the relationship that the reporter has with the youngster but according to the ethnicity and cultural background of the reporter and the child and the cultural context in which the reporter's evaluation occurs.

\section{About the Authors}

MARIEVA PUIG, MA, is a doctoral candidate in child clinical psychology in the
Department of Psychology at Michigan State University. Her current research interests include the development of assessment and prevention strategies that are sensitive to the needs of culturally diverse and underserved populations, adult attitudes toward child psychopathology, and child sexual abuse among ethnic minorities. She is currently completing her internship training at the University of South Florida, Louis de la Parte Florida Mental Health Institute. MICHAEL CANUTE LAMBERT, $\mathrm{PhD}$, is a professor in the Department of Psychology and an adjunct professor at the David Walker Research Institute, College of Human Medicine, at Michigan State University. His research interests include taxonomy and measurement of psychopathology in children and families of the African diaspora, cross-national research on child and adolescent psychopathology, and adult attitudes and behavior regarding child behavior and emotional problems. GEORGE T. ROWAN, PhD, is a professor in the Department of Resource Development in the College of Agriculture and Natural Resource and director of the David Walker Research Institute in the College of Human Medicine at Michigan State University. The Institute's mission is to conduct research on health-related issues that involve policy development directly affecting the lives of African American men in particular and African Americans in general. TYRONE WINFREY, MA, is assistant director at the Office of Admissions at the University of Michigan. His current professional work and interests include facilitating the transition from secondary education into higher education among ethnic minority youth, and nationwide recruitment of ethnic minority youth into college. MIKHAIL. LYUBANSKY, MA, is a clinical doctoral candidate in the department of psychology at Michigan State University. His research interests include cross-national differences in child and adolescent behaviors and the process of acculturation across the lifespan. $\mathrm{He}$ is currently completing his internship training, with an emphasis on multicultural counseling, at the MSU Counseling Center. STANLEY D. HANNAH, MEd, is a doctoral candidate at clinical psychology at the Curry School of Education, University of Virginia. His current research interest is selfesteem and delinquency in ethnic minority youth, and his clinical interests include the assessment of violent youths and counseling minority youths and families. He is currently working on the Virginia Youth Violence Project at the University of Virginia. MARTIN F. HILL, MA, is a doctoral candidate in 
counseling psychology in the College of Education at Michigan State University. His current research interest focus on resiliency and adolescent behavior among African Americans. His clinical interests include adolescent adjustment and coping strategies used after traumatic experiences. Address: Marieva Puig, The Louis de la Parte Florida Mental Health Institute, University of South Florida, 13301 Bruce B. Downs Blvd., Tampa, FL 33612-3807.

\section{Authors' Notes}

1. This study was supported through a grant from the David Walker Research Institute at Michigan State University and a Minority Research Training Grant from the National Institute of Mental Health, and the Institute of International Health, which we acknowledge with gratitude.

2. We thank Valarie François-Bellas, Todd C. Roy, Beth Milburn, Jennifer Carwell, Faith Markle, and Jieun Lee for their support and assistance with data collection and reduction. Finally, we thank the staff and teachers who participated in the project for the time they invested.

\section{References}

Achenbach, T. M. (1986). The child behavior checklist-Direct observation form. Burlington: University of Vermont, Department of Psychiatry.

Achenbach, T. M. (1991). Manual for the Teacher's Report Form and 1991 Profile. Burlington: University of Vermont, Department of Psychiatry.

Achenbach, T. M., Hensley, V. R., Phares, V., \& Grayson, D. (1990). Problems and competencies reported by parents of Australian and American children. Journal of Child Psychology and Psychiatry, 31, 265-286.

Achenbach, T. M., Verhulst, F. C., Baron, G. D., \& Akkerhuis, G. W. (1987). Epidemiology comparisons of American and Dutch children: I. Behavioral/emotional problems and competencies reported by parents for ages 4 to 16. Journal of the American Academy of Child and Adolescent Psychiatry, 26, 317-325.

Cliff, N. (1987). Analyzing multivariate data. Orlando, FL: Harcourt Brace Jovanovich.
Cohen, J. (1988). Statistical power analyses for the behavioral sciences (2nd ed). New York: Academic Press.

Dohrenwend, B. P., Levav, I., Shrout, P. E., Shwartz, S., Naveh, G., Link, B. G., Skodol, A. E., \& Stueve, A. (1992). Socioeconomic status and psychiatric disorders: The causation-selection issue. Science, 255, 946-952.

Hollingshead, A. B. (1975). Four factor index of social status. New Haven, CT: Yale University, Department of Sociology.

Lambert, M. C., Knight F. H., \& Costigan, C. L. (1994). Behavior profile of a psychiatric screening instrument for Jamaican children aged 6-11. International Journal for Intercultural Relations, 18, 507-519.

Lambert, M. C., Knight, F. H., Taylor, R., \& Achenbach, T. M. (1994). Epidemiology of behavioral and emotional problems among children of Jamaica and the United States: Parent reports for ages 6-11. Journal of Abnormal Child Psychology, 22, 113-128.

Lambert, M. C., Knight, F. H., Taylor, R., \& Achenbach, T. M. (1996). Comparison of behavioral and emotional problems among children of Jamaica and the United States: Teacher reports for ages 6-11. Journal of Cross-Cultural Psychology, 27(1), 83-97.

Lambert, M. C., Lyubansky, M., \& Achenbach, T. M. (1998). Behavioral and emotional problems among adolescents of Jamaica and the United States: Parent, teacher, and self-reports for ages 12 to 18 . Journal of Emotional and Behavioral Disorders, 6, 180-187.

Lambert, M. C., Puig, M., Rowan, G. T., Lyubansky, M., \& Winfrey, T. (1998). Adult perspectives on behavior and emotional problems in African-American children. Manuscript submitted for publication.

Lambert, M. C., Weisz, H. R., Knight, F., Desrosiers, M. F., Overly, K., \& Thesiger, C. (1992). Jamaican and American perspectives on child psychopathology: Further exploration of the threshold model. Journal of Consulting and Clinical Psychology, 60, 146-149.

Malgady, R. G., \& Rogler, L. H. (1988). Reply to "The empirical basics of ethnocultural and linguistic bias in mental health." Evaluations of Hispanics. American Psychologist, 43, 1097.

McLoyd, V. C. (1995). Methodological and conceptual issues in the study of children of color. In H. E. Fitzgerald, B. E. Lester, \& B. Zuckerman (Chairs), Children of Color: Research, health, and public policy issues. Round table conducted at the biennial meeting of the Society for Research in Child Development, Indianapolis, IN.

Neter, J., Wasserman, W., \& Kutner, M. H. (1985). Applied linear statistical models (2nd ed.). Homewood, IL: Irwin.

Reed, M. L., \& Edelbrock, C. (1983). Reliability and validity of the Direct Observation Form of the Child Behavior Checklist. Journal of Abnormal Child Psychology, 11, 521-530.

Smith, A. G. (1984). Culture, race, and class in the Commonwealth Caribbean. Mona, Jamaica: University of West Indies, Department of Extramural Studies.

Sylva, K. (1994). School influences on children's development. Journal of Child Psychology and Psychiatry and Allied Disciplines, 35, 135-170.

Weisz, J. R., Chaiyasit, W., Weiss, B., Eastman, K. L., \& Jackson, E. W. (1995). A multimethod study of problem behavior among Thai and American children in school: Teacher reports vs. direct observations. Child Development, 66, 402-415.

Weisz, J. R., Sigman, M., Weiss, B., \& Mosk, J. (1993). Parent reports of behavioral and emotional problems among children in Kenya, Thailand, and the United States. Child Development, 64, 98-109.

Weisz, J. R., Suwanlert, S., Chaiyasit, W., Weiss, B., Achenbach, T. M., \& Trevathan, D. (1988). Epidemiology of behavioral and emotional problems among Thai and American children: Teacher reports for ages 6-11. Journal of Child Psychology and Psychiatry, 30, 471-484.

Weisz, J. R., Suwanlert, S., Chaiyasit, W., Weiss, B., Achenbach, T. M., \& Walter, B. A. (1987). Epidemiology of behavioral and emotional problems among Thai and American children: Parent reports for ages 6-11. Journal of the American Academy of Child and Adolescent Psychiatry, 26, 890-898.

Zimmerman, R. S., Khoury, E. L., Vega, W. A., Gil, A. G., \& Warheit, G. J. (1995). Teacher and parent perceptions of behavior problems among a sample of African American, Hispanic, and non-Hispanic white students. American Journal of Community Psychology, 23, 181-196. 